MEG/EEG study of children with benign partial epilepsy, reported a correlation between location of focal interictal spikes and selective cognitive deficits. Massa R et al (Neurology 2001;57:1071-1079) found that EEG interictal patterns and their persistence were the hallmarks of neuropsychological impairments in 10 of 35 patients $(28 \%)$ with BECTS followed from onset to complete recovery. While these studies focused on the correlation between epileptiform discharges and cognitive impairments in children with BECTS, the current study from Brazil notes a relation between alterations in amplitude of background EEG activity and educational problems.

Ictal EEG and BECTS. Tedrus et al, the Brazil research team, also report details of an ictal EEG during oropharyngeal seizures in an 8-year-old boy with BECTS (Clinical EEG and Neuroscience July 2009;40(3):200-203). Seizure onset during sleep correlated with an increase in centrotemporal spikes followed by slow waves in the right hemisphere. A brief decrease in amplitude of background activity preceded rhythmic, diffuse sharp waves in the right centrotemporal region. Finally, high potential spikes reappeared in the central and temporal regions of the right hemisphere with normalization of background activity. The EEG changes occurred concurrently with clonic lip movements, pouting, and throat noises.

\title{
COGNITIVE DEFICITS AT ONSET OF EPILEPSY
}

Neuropsychological functioning and academic achievement were determined at the time of the first seizure in a prospective study of 282 children (ages 6-14 years, with IQ > 70) and compared with 147 healthy siblings examined at Indiana University, Case Medical Center, and Cincinnati Children's Hospital. Children with seizures scored lower than siblings on all neuropsychological tests, especially involving attention/executive/construction, but academic achievement was unaffected. Neuropsychological deficit was exhibited by $27.4 \%$ of children with seizures vs $18.2 \%$ of healthy siblings $(\mathrm{p}=0.04)$. Almost twice as many with seizures showed deficits in attention/executive/construction, verbal memory and learning, language and processing speed. Only symptomatic/cryptogenic seizure etiology was a risk factor for cognitive deficit. Children taking valproic acid scored lower for processing speed vs no-AED group $(\mathrm{p}=0.009)$. Among children with seizures, those on AEDs performed worse than no-AED group on all neuropsychological factors, but not on academic achievement. EEG epileptiform activity was associated with slower processing speed $(\mathrm{p}=0.004)$ but not with academic achievement. Risk factors for cognitive deficits were multiple seizures, AEDs, symptomatic/cryptogenic etiology, and epileptiform activity on initial EEG; a child with all 4 risk factors is 3 times more likely than healthy siblings to have neuropsychological deficits by the first clinic visit. Absence epilepsy was an added risk factor. (Fastenau PS, Johnson CS, Perkins SM, et al. Neuropsychological status at seizure onset in children. Risk factors for early cognitive deficits. Neurology Aug 12, 2009;73:526-534). (Respond: Philip S Fastenau $\mathrm{PhD}$, Department of Neurology, University Hospitals Case Medical Center, 11100 Euclid Ave, HAN 5040, Cleveland. OH 44106. E-mail: Philip.Fastenau@uhhospitals.org).

COMMENT. Children identified with a first seizure exhibit neuropsychological deficits in $27 \%$ cases. In those with risk factors, up to $40 \%$ have cognitive deficits. Children with multiple seizures, symptomatic/cryptogenic seizure etiology, those on AEDs, or with epileptiform discharges on the initial EEG should be referred for neuropsychological and educational evaluation. 